\title{
Localization properties of the topological charge density and the low lying eigenmodes of overlap fermions
}

\author{
Yoshiaki Koma* \\ Deutsches Elektronen-Synchrotron DESY, 22603 Hamburg, Germany \\ E-mail: yoshiaki.koma@desy.de
}

\section{Ernst-Michael IIgenfritz}

Institut für Physik, Humboldt Universität zu Berlin, 12489 Berlin, Germany

E-mail: ilgenfriephysik.hu-berlin.de

\section{Karl Koller}

Sektion Physik, Universität München, 80333 München, Germany

E-mail: karl.kollerelrz.uni-muenchen.de

\section{Gerrit Schierholz}

Deutsches Elektronen-Synchrotron DESY, 22603 Hamburg, Germany

John von Neumann-Institut für Computing NIC, DESY, 15738 Zeuthen, Germany

E-mail: gerrit.schierholz@desy.de

\section{Thomas Streuer and Volker Weinberg}

John von Neumann-Institut für Computing NIC, DESY, 15738 Zeuthen, Germany Institut für Theoretische Physik, Freie Universität Berlin, 14196 Berlin, Germany

E-mail: thomas.streuer@desy.de, volker.weinberg@desy.de

\begin{abstract}
Overlap fermions, which preserve exact chiral symmetry on the lattice, provide a powerful tool for investigating the topological structure of the vacuum. Applying this formulation to zerotemperature quenched SU(3) configurations generated by means of the Lüscher-Weisz action, we define the topological charge density with and without UV filtering and study its properties by looking at the density profile and the two-point correlation function. We observe that the density possesses global sign coherent structures, which get increasingly tangled as more and more modes are included. This change of the structure is also detected by the increasing negative tail of the two-point function. We also study the inverse participation ratio of the eigenmodes and discuss their dimensionality.
\end{abstract}

XXIIIrd International Symposium on Lattice Field Theory

25-30 July 2005

Trinity College, Dublin, Ireland

*Speaker. 


\section{Introduction}

Topological excitations, phenomenologically modeled as an interacting ensemble of instantons and anti-instantons, are believed to play a prominent role in the low-energy behavior of QCD, especially in realizing the axial $\mathrm{U}(1)$ anomaly (large $\eta^{\prime}$ mass) and chiral symmetry breaking. It is thus of great interest to understand the topological structure of the QCD vacuum from first principles and to clarify whether instantons are indeed providing a realistic description.

For this purpose, we employ the overlap fermion Dirac (Neuberger-Dirac) operator defined by

$$
D=\frac{\rho}{a}\left(1+\frac{X}{\sqrt{X^{\dagger} X}}\right), \quad X=D_{W}-\frac{\rho}{a},
$$

where $D_{W}$ is the Wilson-Dirac operator $(r=1.0)$. We set $\rho=1.4$ as the optimal choice. Overlap fermions possess exact chiral symmetry on the lattice and provide $n_{-}+n_{+}$exact zero modes, $D \psi_{n}^{ \pm}=0$, with $n_{-}\left(n_{+}\right)$being the number of modes with negative (positive) chirality $\gamma_{5} \psi_{n}^{-}=$ $-\psi_{n}^{-}\left(\gamma_{5} \psi_{n}^{+}=+\psi_{n}^{+}\right)$. The index is given by $Q=n_{-}-n_{+}$. The non-zero modes with eigenvalue $\lambda, D \psi_{\lambda}=\lambda \psi_{\lambda}$, occur in complex conjugate pairs $\lambda$ and $\lambda^{*}$ and satisfy $\left(\psi_{\lambda}^{\dagger}, \gamma_{5} \psi_{\lambda}\right)=0$. The topological charge density $q(x)$, which satisfies the index theorem with $Q=\sum_{x} q(x)$, is defined by

$$
q(x) \equiv-\operatorname{Tr}\left[\gamma_{5}\left(1-\frac{a}{2} D(x, x)\right)\right]
$$

where ' $T r$ ' is taken for color and spinor indices. The role of the low-lying Dirac eigenmodes for $q(x)$ is exposed by applying the eigenmode expansion, where the cutoff $\lambda_{\text {cut }}$ implies a kind of UV filtering,

$$
q_{\lambda_{\text {cut }}}(x)=-\sum_{|\lambda| \leq\left|\lambda_{\text {cut }}\right|}\left(1-\frac{\lambda}{2}\right) c_{\lambda}(x),
$$

where $c_{\lambda}(x)=\psi_{\lambda}^{\dagger}(x) \gamma_{5} \psi_{\lambda}(x)$ is the local chirality of the mode with eigenvalue $\lambda$. Note that the UV filtering maintains the index theorem independently of the cutoff such that $Q=\sum_{x} q_{\lambda_{\text {cut }}}(x)$, while controlling the UV fluctuation of the density. This is because the index is computed only from the zero modes which actually occur all with the same chirality.

\section{Observables and results}

In this presentation we study the topological charge density as well as its two-point correlation function. Furthermore we investigate the inverse participation ratio (IPR) of the eigenmodes. We use an ensemble of zero-temperature quenched configurations generated by means of the LüscherWeisz gauge action (see Table 1 and ref. [1]). This action is suitable for topological studies since dislocations are greatly suppressed.

Let us first look at the topological charge density. In Fig. 1, we show the density in a given time slice on a $12^{3} 24$ lattice at $\beta=8.10 .{ }^{1}$ The red (green) color surfaces are the isosurfaces corresponding to $q(x)= \pm 0.0005$. Periodic boundary conditions are imposed. In Fig. 2, we show the same plot for the zero-mode contribution alone and for the full density (no cutoff). It is apparent

\footnotetext{
${ }^{1} 3 \mathrm{D}$ movies available on request.
} 
Table 1: Simulation details: the Lüscher-Weisz gauge action is used

\begin{tabular}{|c|c|cc|c|c|}
\hline$\beta$ & $a[\mathrm{fm}]$ & $V=L^{3} T$ & $\left(\mathrm{fm}^{4}\right)$ & \# of conf. & \# of eigenmodes \\
\hline 8.45 & 0.095 & $12^{3} 24$ & $(3.38)$ & 116 & $O(50)$ \\
\hline 8.45 & 0.095 & $16^{3} 32$ & $(10.6)$ & 267 & $O(140)$ \\
\hline 8.45 & 0.095 & $24^{3} 48$ & $(54.0)$ & 186 & $O(160)$ \\
\hline 8.10 & 0.125 & $12^{3} 24$ & $(10.1)$ & 254 & $O(140)$ \\
\hline
\end{tabular}
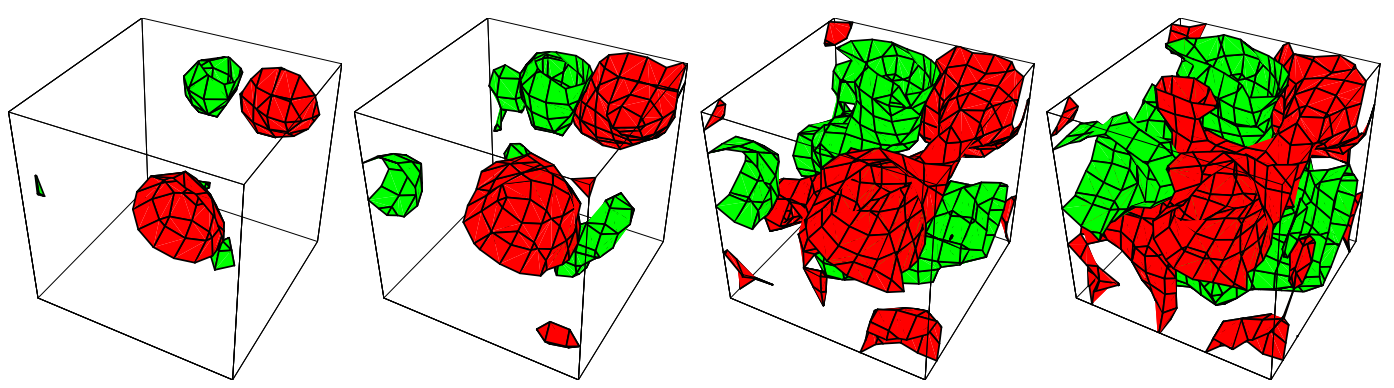

Figure 1: Distribution of the topological charge density in a given time slice of a $12^{3} 24$ lattice at $\beta=8.10$ : cutoff $\operatorname{Im} \lambda_{\text {cut }}=0.14,0.28,0.42,0.56$ (from left to right), isosurfaces at $q(x)= \pm 0.0005$ (red/green).
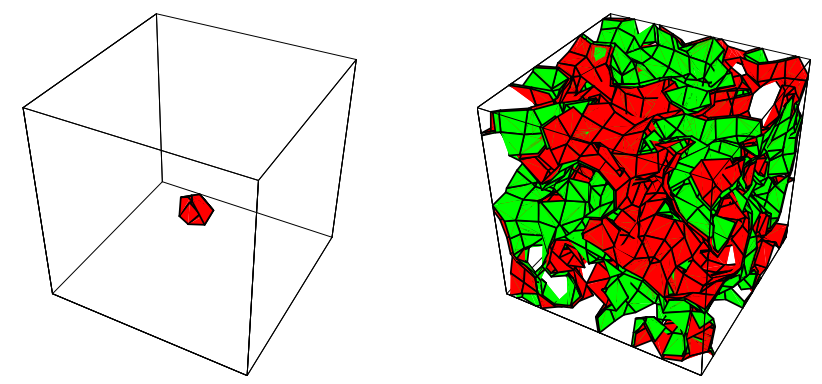

Figure 2: The same plot as in Fig. 1 for the zero mode contribution (left) and the full density (right).

that the topological charge density possesses global sign-coherent structures (clusters). The sizes of the clusters increase as the cutoff $\lambda_{\text {cut }}$ is relaxed. This feature is common also to the other configurations. We will address the local tomography of the clusters of topological charge in a future publication.

Let us next discuss the two-point function of the topological charge density,

$$
C_{q}(R)=\frac{1}{V}\left\langle\sum_{x} q(x) q(y)\right\rangle
$$

where $R=|x-y|$ is the Euclidean distance $(R=r / a)$. The result is shown in Fig. 3. The cutoffs chosen here are corresponding to those in Fig. 1. We observe that while there is always a positive core near the origin at any value of the cutoff, the tail is negative for sufficiently large cutoffs. As the cutoff increases, the peak of the positive core grows and the negative tail also increases. In fact, this is an expected behavior from the density distribution for various cutoffs as seen in Figs. 1 and 2. Since the density of both signs get increasingly tangled when more and more modes are included, any point of positive density comes closer to points of negative density. It is interesting 

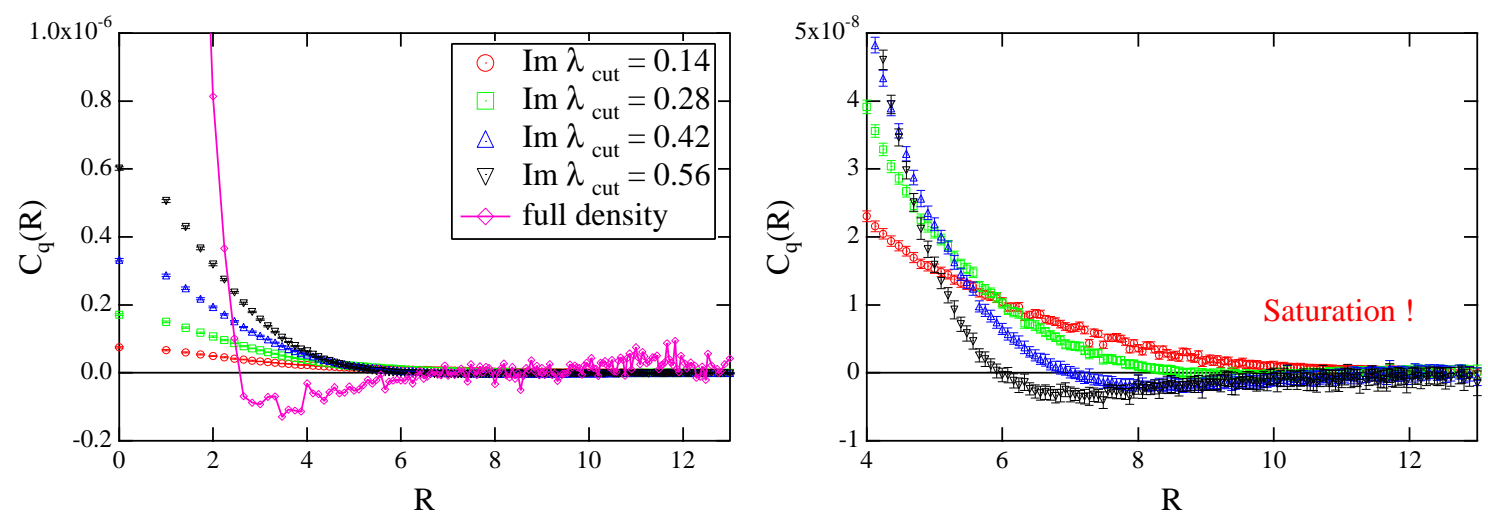

Figure 3: Two-point function of the topological charge density $q_{\lambda_{\text {cut }}}$ on the $12^{3} 24$ lattice at $\beta=8.10$ defined for various $\lambda_{\text {cut }}$, compared to that of the full density $q\left(N_{\text {conf }}=3\right)$. The two-point function is averaged over pairs $(x, y)$ with the same distance $R$. The right figure focusses at the region in $R$ where the cutoff-related correlator turns negative. We obtained a similar picture at $\beta=8.45$.
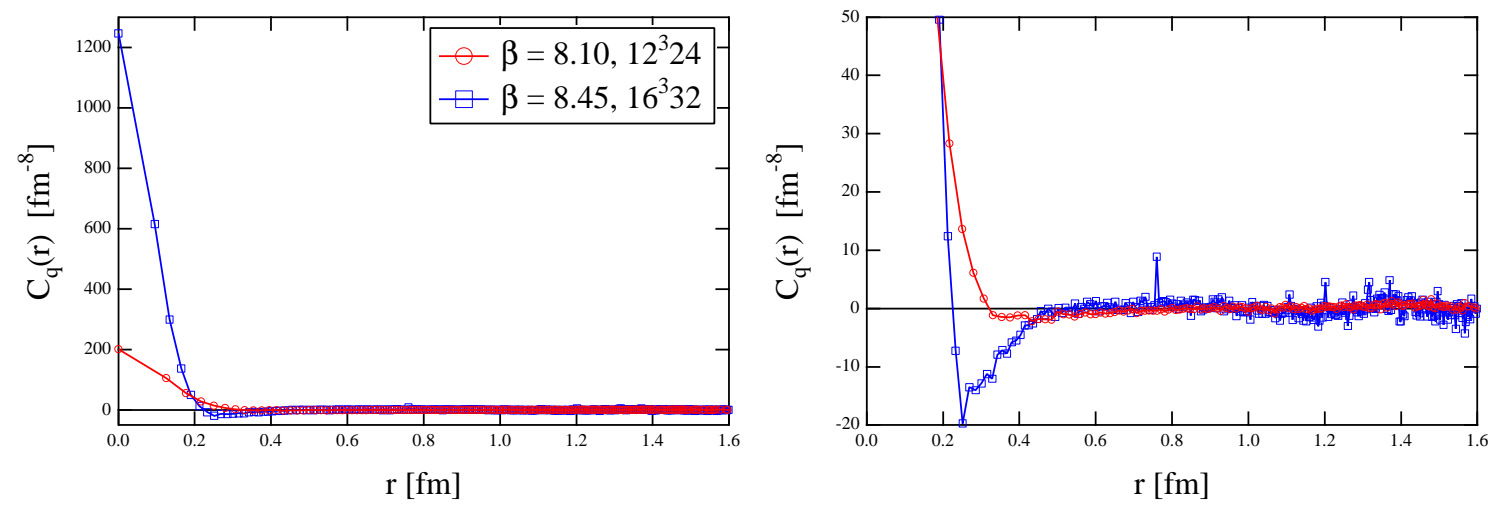

Figure 4: Two-point function of the full topological density on the $12^{3} 24$ lattice at $\beta=8.10\left(N_{\text {conf }}=3\right)$ and on the $16^{3} 32$ lattice at $\beta=8.45\left(N_{\text {conf }}=2\right)$. The right figure shows the same as the left but magnified. Since only a few configurations were available for this comparison we did not estimate the errors.

to note that the topological susceptibility $\chi_{\text {top }}=\left\langle Q^{2}\right\rangle / V$ is not affected by the cutoff. Therefore, as the core peak increases, the contribution from the negative region must grow to maintain the sum over $C_{q}(R)$ with respect to $R$. The dependence on the lattice spacing $a$ is examined in Fig. 4 for the full density, where the scale is introduced by multiplication with $a^{-8}$. Clearly, as $a$ decreases, the core peak (size) is growing (shrinking), which seems to approach an expected behavior in the continuum theory [2], although the finite positive core at our highest $\beta$ stays at a radius $R \approx 2 a$ showing the limits of locality of the operator $q(x)$ itself (see also ref. [3]).

Finally let us discuss the inverse participation ratio (IPR) of the eigenmodes. The IPR of an eigenfunction $\psi_{\lambda}(x)$ is defined by

$$
I=V \sum_{x \in V} \rho_{\lambda}^{2}(x)
$$

where $\sum_{x \in V} \rho_{\lambda}(x)=1$ with $\rho_{\lambda}(x)=\psi_{\lambda}^{\dagger}(x) \psi_{\lambda}(x)$. The IPR, $I=1 / f$, denotes the inverse fraction $f$ of sites forming the support of $\rho_{\lambda}(x)[4,5,6]$. Limiting cases are $I=1$ for $\rho(x)=1 / V$ (delocalized), $I=V$ for $\rho(x)=\delta_{x, x_{0}}$ (extremely localized). From the dependence on $a$ and $V$ one may 

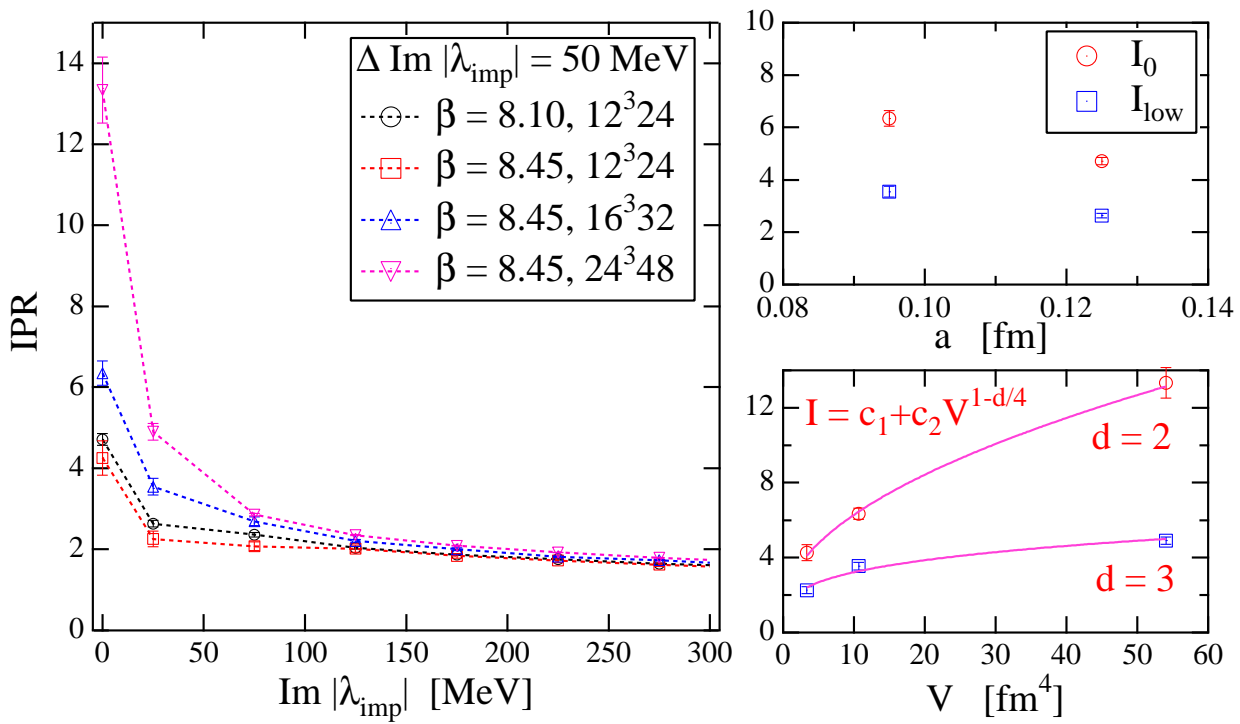

Figure 5: IPR: the dependence on $\operatorname{Im} \lambda$ (left), the $a$ dependence (right upper), and the $V$ dependence (right lower).

infer the effective dimension $d$ of the eigenmode based on the relations $I \propto a^{d-4}$ and $I \propto V^{1-d / 4}$. This means that there is no dependence on $a$ and $V$ if the mode is extending in $d=4$ dimensions. In Fig. 5 (left) we show the IPR as a function of the (imaginary part of the) eigenvalue, where the IPR values are averaged in bins with bin size $\Delta \operatorname{Im} \lambda_{\text {imp }}=50 \mathrm{MeV}$, except for the zero modes which are considered separately. Here, $\lambda_{\text {imp }}$ is the eigenvalue of the improved Dirac operator $D_{\text {imp }}=(1-a D / 2 \rho)^{-1} D$, which projects the eigenvalue of $D$ stereographically onto the imaginary axis. The continuous eigenvalues of $D_{\text {imp }}$ still come in pairs $\pm i \lambda$, while the zero eigenvalues remain zero. In Fig. 5, we also plot the averaged IPR with respect to its $a$ dependence at fixed $V$ (right upper), and with respect to the $V$ dependence with a fixed $a$ (right lower), separately for zero modes $\left(I_{0}\right)$ and lowest non-zero modes $\left(I_{\text {low }}\right)$, the latter for $0<\left|\operatorname{Im} \lambda_{\text {imp }}\right| \leq 50 \mathrm{MeV}$. These plots exhibit that the zero and lowest-lying modes have an IPR clearly larger than one and are sensitive to the change of $a$ and $V$, respectively. This indicates that these modes are localized to some extent and possess an effective dimension less than $d=4$. From the $V$ dependence, $I=c_{1}+c_{2} V^{1-d / 4}$, we find that $d=2$ and $d=3$ describe the IPR data well for the zero and lowest-lying modes, respectively. On the other hand, for the higher modes $\left(\left|\operatorname{Im} \lambda_{\text {imp }}\right|>200 \mathrm{MeV}\right)$ we find $I \lesssim 2$, practically independent on $a$ and $V$. Hence we may conclude that these modes are delocalized and extending in all $d=4$ dimensions. The behavior of the IPR observed here is qualitatively the same as found in the SU(2) case [6]. However, the actual values of the IPR are essentially smaller in our case and our fit results are also different.

\section{Summary}

Overlap fermions preserve exact chiral symmetry on the lattice and provide exact zero modes, which allow us to unambiguously compute the index $Q$ of vacuum configurations. It is then possible to define the topological charge density $q(x)$ and to expose the role of low-lying eigenmodes in $q(x)$ 
by applying the eigenmode expansion (UV filtering). This approach is gauge invariant and leaves the lattice scale unchanged in contrast to the cooling method. In this sense, overlap fermions are a powerful tool for investigating the topological structure of the vacuum.

Using this formalism, we have investigated the localization properties of the topological charge density by looking at the density profile as well as the two-point correlation function on zerotemperature quenched configurations. We have also examined the IPR of the eigenmodes and their dimensionality. We have found that the topological charge density possesses global sign coherent structures, which get increasingly tangled as more and more modes are included. Such a change of density structures has also been detected in the behavior of the two-point function. We note that further preliminary studies show that the full topological charge density has a lower-dimensional laminar structure, together with a lumpy structure inside the sign coherent regions. Details will be presented in our forthcoming publication. The IPR has indicated that the zero and low-lying modes, typically for Im $\lambda_{\text {imp }} \lesssim 200 \mathrm{MeV}$, are localized to some extent and have an effective dimension less than four.

A similar investigation at finite temperature near $T=T_{c}$ on $N_{f}=2$ dynamical gauge configurations is discussed in ref. [7].

\section{Acknowledgments}

The numerical calculations have been performed at NIC (Jülich) and HLRN (Berlin) as well as at DESY (Zeuthen). We thank all institutions for support. This work has been supported in part by the EU Integrated Infrastructure Initiative Hadron Physics (I3HP) under contract RII3CT-2004-506078 and by the DFG under contract FOR 465 (Forschergruppe Gitter-HadronenPhänomenologie).

\section{References}

[1] D. Galletly et al. (QCDSF-UKQCD collaboration), Quark spectra and light hadron phenomenology from overlap fermions with improved gauge field action, Nucl. Phys. B (Proc. Suppl.) 129 (2003) 453 [hep-lat/0310028].

[2] E. Seiler, Some more remarks on the Witten-Veneziano formula for the $\eta^{\prime}$ mass, Phys. Lett. $\mathbf{B 5 2 5}$ (2002) 355 [hep-th/0111125].

[3] I. Horvath et al., The negativity of the overlap-based topological charge density correlator in pure-glue QCD and the non-integrable nature of its contact part, Phys. Lett. B617 (2005) 49 [hep-lat/0504005].

[4] C. Gattringer, M. Göckeler, P.E.L. Rakow, S. Schäfer, and A. Schäfer, Properties of near zero modes and chiral symmetry breaking, Nucl. Phys. B617 (2001) 101 [hep-lat/ 0107016 ].

[5] C. Aubin et al. (MILC collaboration), The scaling dimension of low lying Dirac eigenmodes and of the topological charge density, Nucl. Phys. B (Proc. Suppl.) 140 (2004) 626 [hep-1at / 0410024 ].

[6] F. V. Gubarev, S. M. Morozov, M. I. Polikarpov and V. I. Zakharov, Evidence for fine tuning of fermionic modes in lattice gluodynamics (2005) [hep-lat / 0505016 ].

[7] V. Weinberg et al., Probing the chiral phase transition of $N_{f}=2$ clover fermions with valence overlap fermions, Proceedings of Science (LAT2005) 171 (these proceedings). 